\title{
Estafa Entre Residentes de Cancerologia Clínica: Um Estudo de Coorte Prospectivo de Instituições Brasileiras
}

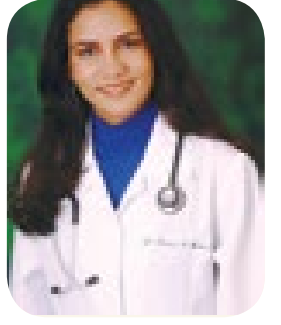

\author{
Daniela Barros ${ }^{1}$, Melba Moura ${ }^{1}$, Rafael Kalii ${ }^{1}$, Larissa Moura ${ }^{1}$ \\ Daniel I. G. Cubero, Renata Rego Lins Fumis; Thiago Hérick de Sá; Aldo Dettino; Felipe Osório \\ Costa; Brigitte M. R. H.; Adam Van Eyll; Carlos Beato; Fernanda Maris Peria; Augusto Mota; \\ José Altino; Sérgio Jobim Azevedo; Duílio Reis da Rocha Filho; Melba Moura; Álvaro Edson \\ Ramos Lessa; Auro del Giglio. Burnout in Medical Oncology Fellows: a Prospective Multicenter \\ Cohort Study in Brazilian Institutions. J Canc Educ.Published online 09 May 2015.
}

\section{RESUMO}

A estafa profissional é frequente entre oncologistas, porém, a ocorrência deste problema entre médicos residentes desta área é pouco estudada, apesar da exposição aos fatores conhecidamente desencadeadores ser a mesma. Este estudo avalia a ocorrência de estafa ao longo do primeiro ano de residência de oncologia clínica em instituições brasileiras.

PALAVRAS-CHAVE: estafa, residência, oncologia.

KEY WORDS: burnout, fellow, medical oncology.

\section{INTRODUÇÃO}

A estafa profissional, tambem conhecida como síndrome de Burnout, resulta do estresse decorrente do ambiente de trabalho e é caracterizada por exaustão emocional, despersonalização (interação com pacientes e pessoas como se fossem objetos) e sentimentos de pouca realização pessoal. ${ }^{1}$

Indivíduos que gerenciam pacientes com doenças crônicas e graves estão em maior risco de desenvolvê-la e a oncologia é um dos campos médicos em que a própria gravidade da doença, o uso de tratamentos tóxicos e prolongados, além do contato próximo com pacientes terminais e seus familiares, podem submeter os profissionais de saúde a estresse considerável. ${ }^{1,2,3}$

A alta prevalência da síndrome de Burnout no ambiente oncológico compromete não apenas a qualidade da vida profissional e pessoal dos envolvidos, mas também a qualidade dos cuidados prestados aos pacientes e seus familiares. ${ }^{2,3}$

A prevalência deste quadro tem sido relatada em índices acima de $40 \%$ em oncologistas e, devido a condições de trabalho semelhantes e exposição aos mesmos fatores estressantes, supõe-se que médicos residentes apresentem índices semelhantes. ${ }^{3}$
Apresentamos um estudo de coorte de médicos recém-admitidos em programas de residência em oncologia clínica, em instituições brasileiras, para identificar incidência e fatores de risco associados a estafa profissional, assim como avaliar a prevalência de depressão e estresse.

\section{MÉTODOS}

Foram selecionados residentes de oncologia de instituições brasileiras filiadas ao sistema público de saúde, de forma que o coordenador dos programas registrados no Brasil em 2009 recebeu convites (por e-mail) para participação e inclusão dos novos residentes nos anos de 2010 e 2011.

A participação no estudo foi facultativa após assinatura de um termo de consentimento e confidencialidade e os dados nao foram disponibilizados individualmente aos coordenadores.

Os voluntários foram solicitados a responder a alguns questionários, contendo informações sociodemográficas, sentimentos e atitudes em relação à síndrome de Bunourt e inventários baseados em níveis de estresse e sintomas depressivos, sendo realizados sequencialmente no início, meio e fim do primeiro ano. Os questionários deveriam ser respondidos individualmente, de forma anônima.

\section{RESULTADOS}

Um total de 54 novos médicos residentes em oncologia clínica de 11 instituições foram alocados no estudo entre 2010 e 2011, com uma média de idade de 28.4 anos. Entre os residentes do primeiro ano não houve diferença entre gêneros, $83.3 \%$ eram solteiros, $94.4 \%$ não tinham filhos e $67.3 \%$ tinham suporte familiar. Grande parte (82.7\%) não praticavam atividade física, nenhum era tabagista e $75 \%$ consumiam 3 ou menos doses de bebida alcoólica por semana. 
Todos os residentes trabalhavam mais que 44 horas or semana, com $55.8 \%$ apresentando carga horária semanal superior a 60 horas, prevista como máxima No ms orientaçoes do Ministerio da Educaçáo.

No momento do ingresso na residência médica, $49 \%$ ja apresentavam níveis elevados de exaustão emocional e $64.7 \%$, altos niveis de despersonalização, chegando a $76 \%$ dos residentes já preencherem critérios para sindrome de Burnout. Entretanto, apenas 4\% apresentavam sinais de depressão moderada a grave $6.1 \%$ apresentavam estresse importante.

A carga de trabalho excessiva, como avaliação de mais de 15 pacientes por dia e/ou mais de 60 horas de trabalho por semana, teve associação significativa com altos niveis dos componentes da síndrome de Burnout, com significância estatística, enquanto a presença de suporte familiar foi fator protetor.

Tabela 1 - Carga de trabalho, prevalência de estafa depressão e estresse entre os residentes egressos nos programas de cancerologia clínica

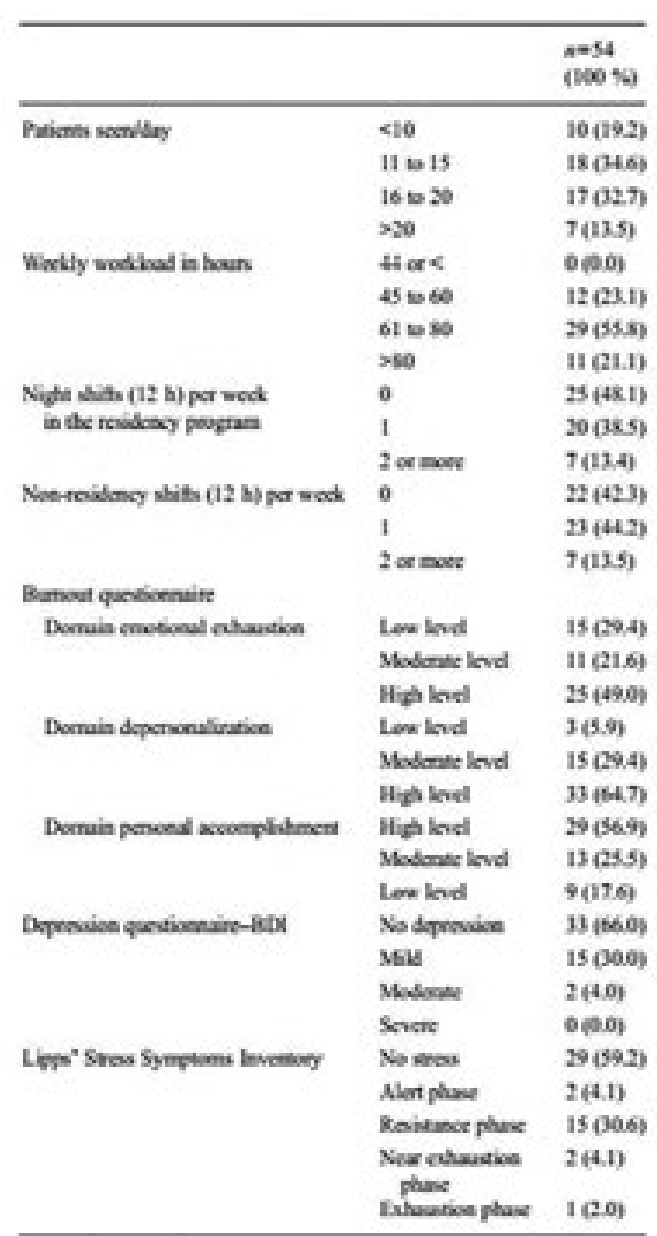

Tabela 2 - Progressão de estafa, estresse e depressão durante o primeiro ano de residência em oncologia clínica

\begin{tabular}{|c|c|c|c|}
\hline & Admission (T1) & End (T3) & $p$ Value \\
\hline 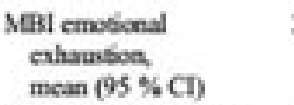 & $25.7(225-28.9)$ & $31.7(29.0-34,4)$ & $0.003^{*}$ \\
\hline $\begin{array}{l}\text { MBSI depersonal inatican. } \\
\operatorname{menn}(95 \% \mathrm{CI})\end{array}$ & $120(10.4-13.6)$ & $145(12.7-16.3)$ & 0.005 \\
\hline 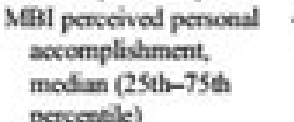 & $41(36-44)$ & $39(35-42)$ & $0.12^{b}$ \\
\hline 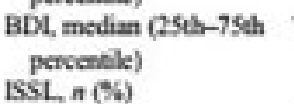 & $7(3-11)$ & $8(4-12)$ & $\begin{array}{l}0.188^{\circ} \\
0.513^{\circ}\end{array}$ \\
\hline No stress & $29(99.2 \%)$ & $24(55.85)$ & \\
\hline Net phax & $2(4.1 \%)$ & $0(0.0)$ & \\
\hline Rosistance phase & $15(30.6 \%)$ & $19(4.2 \%)$ & \\
\hline Near exharition phane & $2(4.1 \%)$ & $0(0.0)$ & \\
\hline Exhaustion phase & $1(2.0 \%)$ & $0(0.0)$ & \\
\hline
\end{tabular}

Mising data for T3 were replaced by the data for T?

-Paired / loot

"Wilkoxon test

"MeNemar's tot (the data were dictotomized in "stress" and "no stress")

\section{DISCUSSÃO}

Este estudo fornece dados epidemiológicos de médicos que iniciam a residência médica em oncologia clínica em instituições brasileiras e demonstra que a maioria desses medicos ja apresentam sintomas de Burnout desde o ingresso na residência, não sendo assim possível identificar os fatores de risco para surgimento desta síndrome.

No entanto, os sintomas se agravaram de forma significativa durante o primeiro ano deste programa, com un aumento da prevalência de 76 para $88 \%$ entre o primeiro e o último questionario do ano, sendo o excesso de carga de trabalho significativamente associado a esta sindrome. Além de interferir na qualidade de vida individual, depressão, estresse e estafa profissional podem afeta a qualidade do cuidado para com o paciente, já tendo sido demonstrado em literatura, num estudo realizado com residentes de pediatria, que a chance de erros de prescrição era 6.2 vezes maior entre residentes que apresentavam depressão.,3,5,

A alta prevalência de estafa entre residentes de oncologia neste estudo mostra um sério problema na qualidade de vida dos médicos e representa um risco potencial para a qualidade do atendimento prestado ao paciente. ${ }^{5,6,7}$
São necessários mais estudos para investigação do tema, assim como a busca de estratégias para prevenção e redução do impacto destes problemas na qualidade da atividade profissional.

\section{REFERÊNCIAS}

1. Ramirez AJ, Graham J, Richards MA, Cull A Gregory WM, Leaning MS, Snashall DC, Timothy AR (1995) Burnout and psychiatric disorder among cancer clinicians. Br J Cancer 71(6): 1263-1269.

2. Whippen DA, Canellos GP (1991) Burnout syndrome in the practice of oncology: results of a random survey of 1,000 oncologists. J Clin Oncol 9 (10): 1916 1920.

3. Trufelli DC, Bensi CG, Garcia JB, Narahara JL, Abrão MN, Diniz RW, Miranda Vda C, Soares HP, Del Giglio A (2008) Burnout in cancer professionals: a systematic review and meta-analysis. Eur $J$ Cancer Care (Engl) 17(6): 524-531. doi: 10.1111/j.1365-2354. 2008. 00927.x.

4. Glasberg J, Horiuti L, Novais MA, Canavezzi AZ, da Miranda Costa V, Chicoli FA, Gonçalves MS, Bensi CG, del Giglio A (2007) Prevalence of the burnout syndrome among Brazilian medical oncologists. Rev. Assoc. Med. Bras. 53 (1): 85-89.

5. Fahrenkopf AM, Sectish TC, Barger LK, Sharek PJ, Lewin D, Chiang VW, Edwards S, Wiedermann $\mathrm{BL}$, Landrigan CP (2008) Rates of medication errors among depressed and burnt out residents: prospective cohort study. BMJ 336 (7642): 488-491. doi: 10. 1136/ bmj. 39469. 763218. BE.

6. West CP, Huschka MM, Novotny PJ, Sloan JA, Kolars JC, Habermann TM, Shanafelt TD (2006) Association of perceived medical errors with resident distress and empathy: a prospective longitudinal study. JAMA 296(9): 1071-1078.

7. Blanchard P, Truchot D, Albiges-Sauvin L, Dewas S, Pointreau Y, Rodrigues M, Xhaard A, Loriot Y, Giraud P, Soria JC, Kantor G (2010) Prevalence and causes of burnout amongst oncology residents: a comprehensive nationwide cross-sectional study. Eur J Cancer46 (15) 2708-2715. doi: 10.1016/j.ejca.2010.05.014.

1- Serviço de Cancerologia Clínica do Hospital Santa Izabel

Endereço para correspondência:

danielagbarros@gmail.com 